\title{
New clues in the COX2 mystery
}

Why do cyclooxygenase-2 (COX2) inhibitors increase the risk of heart attack and stroke? And can such adverse effects be avoided while retaining anti-inflammatory efficacy? Potential answers to both questions are suggested by a recent study from Garret FitzGerald and colleagues published online in the Journal of Clinical Investigation.

The development of selective COX2 inhibitors was initially stimulated by the hypothesis that COX1 was a constitutively expressed isoform whose inhibition could cause the gastrointestinal side effects characteristic of traditional nonsteroidal anti-inflammatory drugs (NSAIDs), whereas COX2 was an inducible isoform whose inhibition results in the anti-inflammatory effects of these drugs. However, the hypothesis seems over-simplistic in the light of the problems with COX2 inhibitors, and considerable attention is now being focused on gaining a deeper understanding of the mechanisms underlying the beneficial and adverse effects of COX inhibition.

A key to understanding these effects seems to be the relative influence of COX1 and COX2 inhibition on the levels of the various prostanoids that result from further enzymatic modification of the product of both COX enzymes, prostaglandin $\mathrm{H}_{2}$. For example, suppression of the production of the prostanoid prostaglandin $\mathrm{E}_{2}$ is believed to underlie the anti-inflammatory efficacy of COX2 inhibitors. However, it has been proposed that concomitant alterations of the levels of other prostanoids - thromboxane $A_{2}$, which has prothrombotic effects, and prostacyclin, which has

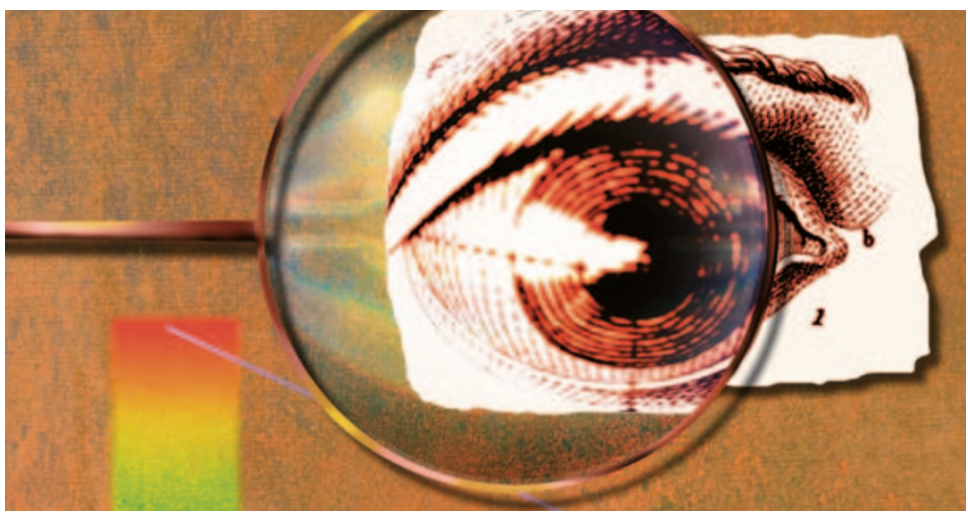

antithrombotic effects - might cause cardiovascular side effects.

To investigate the importance of the levels of various prostanoids, and the effects of COX inhibition on them, FitzGerald et al. used several mouse models with knockout, knockdown or mutation of one or both of the COX enzymes, and also selective COX2 inhibitors. These experiments provided evidence that COX2 is the dominant source of prostacyclin in vivo, as is COX1 for thromboxane $\mathrm{A}_{2}$, supporting the proposal that disruption of the balance between prostacyclin and thromboxane $\mathrm{A}_{2}$ could underlie the cardiovascular problems associated with selective COX2 inhibition. Indeed, inhibition, deletion or inactivation of COX2 augmented the response to thrombogenic stimuli and also elevated blood pressure, and these responses were attenuated by knockdown of COX1, which mimics the known antithrombotic effects of low-dose aspirin.

So, is there any way to obtain the beneficial anti-inflammatory effects of COX2 inhibition without increasing the risk of serious cardiovascular events? Intriguingly, a previous study had shown that deletion of microsomal PGE synthase 1 (mPGES1), which synthesizes prostaglandin $\mathrm{E}_{2}$ from prostaglandin $\mathrm{H}_{2}$, is as effective as traditional NSAIDS in models of pain and inflammation, and so the authors investigated the effects of mPGES1 deletion further. Prostaglandin $\mathrm{E}_{2}$ was depressed, prostacyclin was augmented, there was no effect on thromboxane $\mathrm{A}_{2}$ and, most importantly, mPGES1 deletion did not affect either thrombogenesis or blood pressure. Taken together, these observations suggest that inhibitors of mPGES1 could retain the anti-inflammatory effects of COX2 inhibitors while being less prone to their adverse cardiovascular consequences, and so might represent a promising focus for future drug development efforts.

Peter Kirkpatrick

ORIGINAL RESEARCH PAPER Cheng, Y. et al. Cyclooxygenases, microsomal prostaglandin $\mathrm{E}$ synthase-1, and cardiovascular function. J. Clin. Invest. 116, 1391-1399 (2006)

FURTHER READING Mitchell, J. A. \& Warner, T. D. COX isoforms in the cardiovascular system: understanding the activities of non-steroidal anti-inflammatory drugs. Nature Rev. Drug Discov. 5, 75-86 (2006) | Grosser T., Fries, S. \& FitzGerald, G. A. Biological basis for the cardiovascular consequences of $\mathrm{COX}-2$ inhibition: therapeutic challenges and opportunities. J. Clin. Invest. 116, 4-15 (2006) 